\title{
ANÁLISIS EXPLORATORIO DE LOS CUIDADOS AL PARTO EN NICARAGUA DESDE EL MARCO DE LOS DERECHOS SEXUALES Y REPRODUCTIVOS
}

\author{
EXPLORATORY ANALYSIS OF CHILDBIRTH CARE IN NICARAGUA FROM \\ THE FRAMEWORK OF SEXUAL AND REPRODUCTIVE RIGHTS
}

Recibido: 04/05/2018 - Aceptado: 18/05/2018

\begin{abstract}
Resumen
Con el objetivo de explorar la calidad de los cuidados al parto y el ejercicio de violencia obstétrica durante el parto, esta investigación analizó experiencias de parto recogidas a través de un cuestionario aplicado en 2013 a 24 mujeres. A través de los indicadores analizados, se comprueba cómo los cuidados al parto siguen sin incorporar los máximos estándares de calidad, ignorando la evidencia científica y violando los derechos a la salud de las mujeres: el $89 \%$ de las participantes declaró no haber ejercido el consentimiento informado. Este estudio confirma el incumplimiento de las recomendaciones de la Organización Mundial de la Salud para la atención al parto y la violación de los derechos humanos de las mujeres cuando paren en Nicaragua.
\end{abstract}

Palabras clave: Violencia obstétrica; tasa de cesáreas; cuidados al parto; derechos sexuales y reproductivos; experiencias de parto.

\begin{abstract}
With the objective of exploring the quality of delivery care and the exercise of obstetric violence during childbirth, this research analyzed women's delivery experiences. The primary data were collected through a questionnaire applied in 2013 to 24 women. Through the analyzed indicators, it is verified that the care during labor and delivery still do not incorporate the quality standards, ignoring the scientific evidence and violating women's health rights: $89 \%$ declared not having exercised the informed consent. This study confirms the unsatisfactory fulfillment of the recommendations of the $\mathrm{WHO}$ for the care of childbirth and the violation of the human rights of the women in the framework of the care of the childbirth in Nicaragua.
\end{abstract}

Keywords: Obstetric violence; cesarean rates; delivery care; sexual and reproductive rights; childbirth experiences. 


\section{Introducción $^{1}$}

Entre las dificultades de las mujeres para acceder a un desarrollo humano pleno en el siglo XXI, muchos países siguen tropezando con la misma piedra: el ejercicio de los derechos sexuales y reproductivos de las mujeres (en adelante DSR). Nicaragua no es una excepción. En el punto de mira político, académico y social, las cifras de mortalidad materna, la prohibición absoluta del aborto y la problemática de los embarazos adolescentes son temas habituales y de gran controversia en el debate social.

Así mismo, para los especialistas en salud materna son bien conocidas las sobretasas de cesáreas de Nicaragua, presentes en todos los sistemas de atención que forman parte del sistema nacional de salud. Si la tasa normal de cesáreas oscila entre el 5 por ciento y el 15 por ciento a nivel poblacional (Gibbons et al. 9), en Nicaragua las tasas más contenidas -las que ofrece el sistema público del sector salud- oscilaron entre el 26 por ciento y el 34 por ciento en el periodo 2006-2012 (Instituto Nacional de Información de Desarrollo (INIDE), 2013). Cada cierto tiempo, la prensa nacional señala que las tasas de cesáreas siguen siendo muy superiores a las orientadas por la Organización Mundial de la Salud (OMS). Desde el ámbito clínico, en Nicaragua este fenómeno ha sido relacionado con una supuesta mayor demanda por parte de las mujeres y con la existencia de un esquema de incentivos perverso que conduce a un incremento de los ingresos financieros de los actores privados y a la ineficacia en la gestión del gasto público. Por el contrario, es poco habitual que los actores implicados se detengan en el análisis del problema que se esconde detrás de la sobretasa de cesáreas: el ejercicio de los DSR de las mujeres directamente vinculados con la vivencia de la maternidad.

En este artículo presento los resultados y conclusiones de una investigación que profundiza en las causas que vendrían a explicar la sobretasa de cesáreas desde la perspectiva de las mujeres con experiencias de parto y cesárea. La investigación, de carácter exploratorio, se llevó a cabo con el objetivo de analizar la calidad de los cuidados que reciben las mujeres durante el parto y la cesárea dentro del sistema de salud de Nicaragua. ${ }^{2}$

\section{Antecedentes}

\section{Comportamiento de la tasa de cesáreas en Nicaragua como indicador de calidad y resultado de la atención al parto}

Como se ha apuntado anteriormente, a nivel poblacional los mejores resultados, en términos de mortalidad y morbilidad materna y neonatal, se observan cuando la tasa de cesáreas oscila entre el 5 por ciento y el 15 por ciento. Cuando los partos por cesárea son superiores al 15 por ciento o inferiores al 5 por ciento, es indicativo de que 
en el sector salud existen factores (relacionados con la práctica clínica o no) que están impactando negativamente sobre la calidad de los cuidados a la salud durante el parto y sobre los resultados en términos de salud materna y neonatal (Villaverde 31).

En Nicaragua, los últimos datos disponibles de la Encuesta Nicaragüense de Demografía y Salud (ENDESA) indican que, en promedio, para el periodo 2006-2012, la tasa de cesáreas alcanzó el 32 por ciento para el conjunto del sistema de salud; un 28 por ciento para el régimen no contributivo, ${ }^{3}$ un 54 por ciento para el régimen contributivo y el 69 por ciento para el régimen de financiación privada ${ }^{4}$ (Figura 1) (INIDE 2013). Es decir, en el mejor de los casos, la tasa de cesáreas en Nicaragua dobla a la tasa máxima esperada.

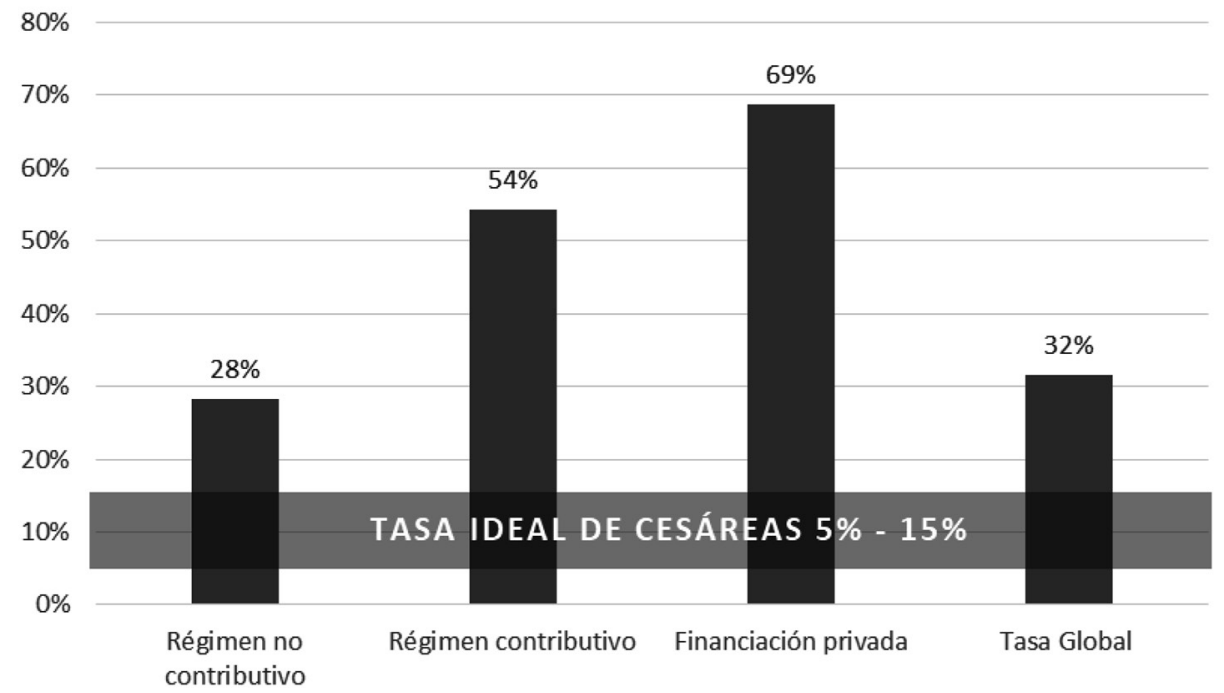

Fuente: Elaboración propia a partir de INIDE (2013).

La diferencia de tasas, según el régimen de financiación, apunta al peso de los distintos esquemas de incentivos en relación con la decisión de realizar una cesárea. Posiblemente, también muestre diferencias en el grado y en los tipos de violencia ejercida contra las mujeres durante el embarazo y en el parto.

Al analizar la evolución de la tasa en el periodo 2006-2012, se puede observar que la sobretasa de cesáreas es estructural para el sistema de salud (Figura 2). Para el régimen contributivo y el sistema de financiación privada, se observa una reducción importante en la tasa a partir del 2010, la cual podría estar relacionada con los cambios 
normativos introducidos a partir del 2008, especialmente con la introducción de la Norma 042 para la Humanización del Parto Institucional (Ministerio de Salud de Nicaragua).

Figura 2

EVOLUCIÓN TASA DE CESÁREAS ENDESA POR RÉGIMEN DE FINANCIACIÓN. PERIODO 2006-2012

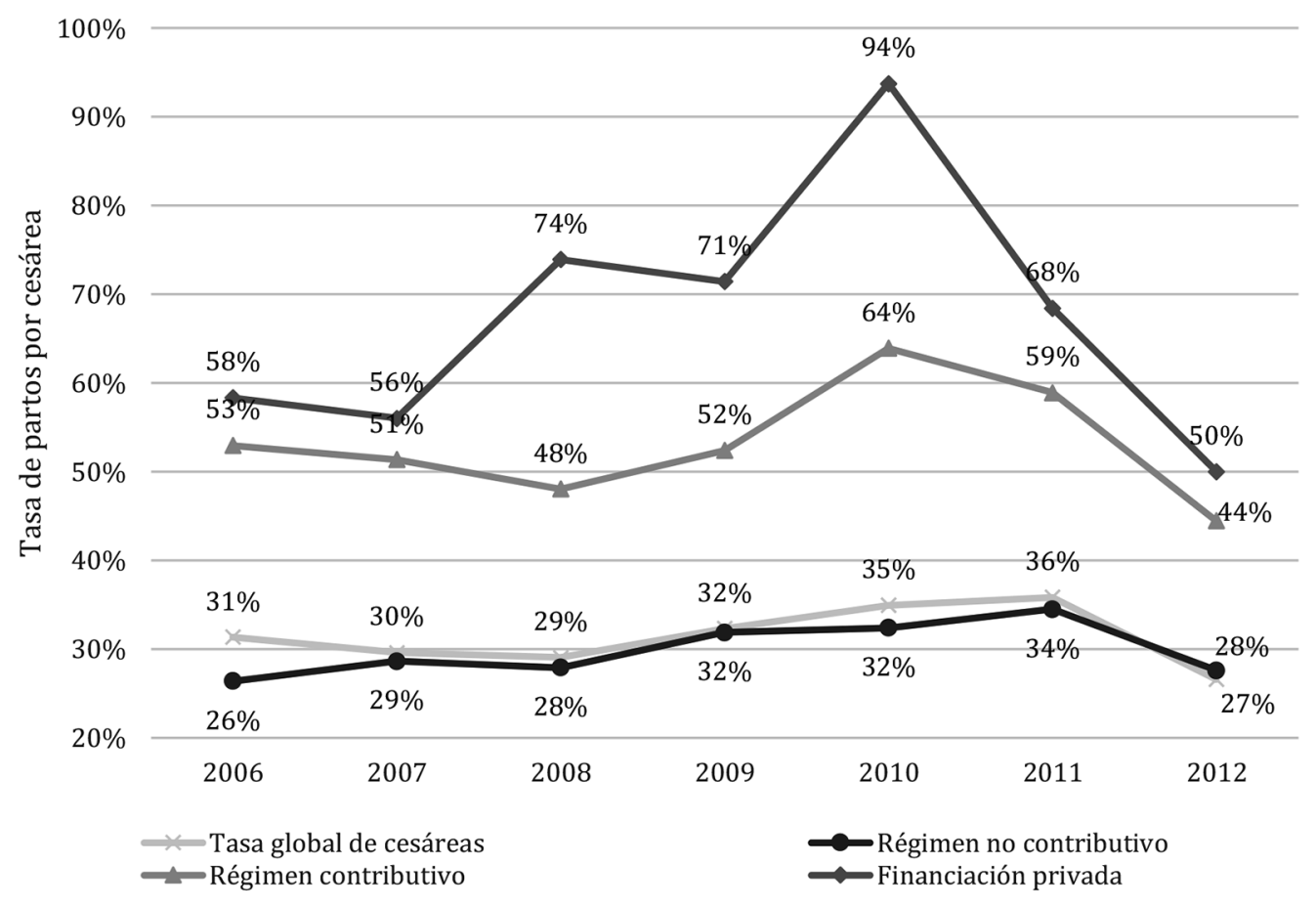

Fuente: Elaboración propia a partir de INIDE (2013).

Sin embargo, las estadísticas que publica anualmente el Instituto Nacional de la Seguridad Social para los partos atendidos dentro del régimen contributivo (Figura 3) indican que para este régimen, el impacto de la reforma en el marco regulatorio fue reducido en cuanto a la tasa de cesáreas y no se ha sostenido en el tiempo, pues a partir del año 2012 las tasas volvieron a crecer por encima de los niveles previos al 2008. 
Figura 3

EVOLUCIÓN TASA DE CESÁREAS INSS PARA EL RÉGIMEN CONTRIBUTIVO

POR TIPO DE PARTO. PERIODO 2005-2015

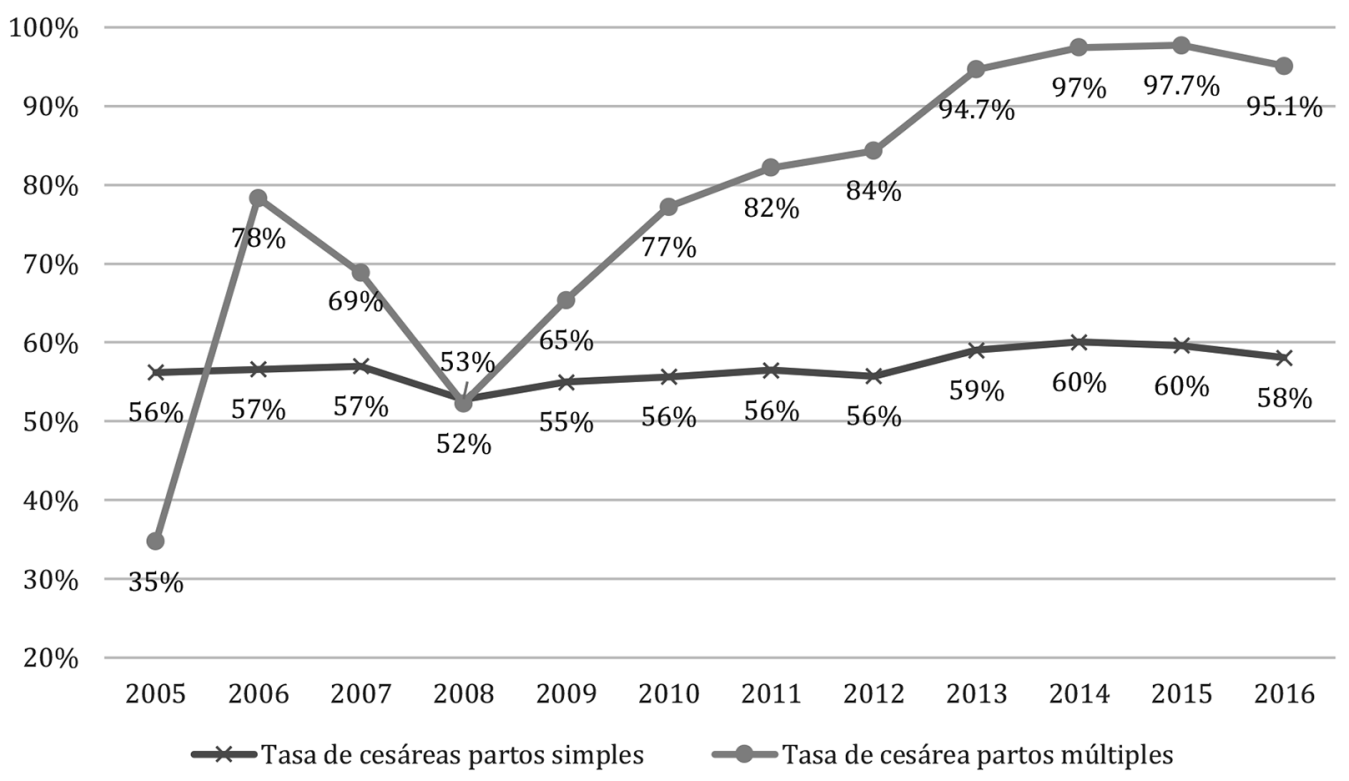

Fuente: Elaboración propia a partir de estadísticas del INSS $(2015,2016)$.

En conclusión, para todos los regímenes de financiación las tasas de cesáreas son muy superiores al límite superior de la tasa ideal. Este comportamiento de la tasa de cesáreas, el cual es estructural para todo el sistema de salud, cuestiona la calidad de los cuidados al parto recibidos por las mujeres (Caño 3) e incrementa los riesgos para la salud de las mujeres y las criaturas recién nacidas: para las mujeres, la cesárea incrementa el riesgo de mortalidad materna en más del doble, además de incrementar el riesgo de sufrir complicaciones diversas, entre ellas la infección puerperal, hemorragia, tromboembolismo, lesión de órganos próximos (como la vejiga o los intestinos), histerectomía, síndrome postraumático y depresión postparto, además de generar repercusiones negativas a largo plazo en los futuros embarazos, como un mayor riesgo de acretismo, rotura uterina, placenta previa o embarazos ectópicos (Villaverde 29; Lumbiganon 496; Coalition for Improving Maternity Services 1-3).

Para las criaturas recién nacidas, la cesárea también presenta mayores riesgos, mostrando test de Apgar con puntuaciones un 50 por ciento más bajas, un riesgo 5 veces mayor de ingreso o de necesitar asistencia respiratoria, de sufrir una lesión por el uso del bisturí o de menor prevalencia de la lactancia materna y las consecuencias negativas asociadas a ello (ídem). A largo plazo, para la criatura recién nacida, el nacimiento por 
cesárea se asocia con un riesgo mayor de padecer obesidad, asma, reacciones alérgicas y autismo (Sáez 4; Odent 57; Curran et al. 937; Polidano et al. 1).

\section{Factores causales vinculados a la sobretasa de cesáreas}

Qué factores explican el comportamiento de la tasa de cesáreas en Nicaragua es un dato que actualmente se desconoce. En una investigación de tipo cualitativo realizada en el 2014 en Nicaragua, se analizaron estos factores, siendo agrupados en dos categorías: factores clínicos y no clínicos (Colomar et al. 2384). En este estudio, en el cual participaron 17 especialistas en activo en obstetricia o ginecología y 9 personas con responsabilidades en la toma de decisiones en el sector salud, se identificaron una gran variedad de factores no clínicos que están presionando al alza la tasa de cesáreas, entre los que destacaron los factores culturales. El equipo de investigación verificó en esta población de estudio la permanencia de la creencia según la cual la sobretasa de cesáreas está vinculada con los factores económicos y la presión que ejercen las mujeres y sus familias para que los nacimientos tengan lugar por cesárea. Sin embargo, el estudio no incorporó las percepciones ni las experiencias de las mujeres, ni tampoco analizó la tasa de cesáreas en relación con la calidad de los cuidados al parto, por lo que sus conclusiones no permiten entender el problema de la sobretasa de cesáreas en su globalidad.

En relación a los factores clínicos, los estudios médicos indican que en la actualidad, el diagnóstico de prolongación o detención de la primera y segunda fases del parto (dilatación y expulsivo) están entre las indicaciones principales para la cesárea primaria (OMS 2015 3), siendo la primera indicación para la cesárea no programada en EE. UU. (Dekker 1-2). En su guía de cuidados al parto normal de 1996, la OMS vinculaba el fracaso en el progreso de parto con los cuidados inadecuados facilitados a las mujeres. Los cuidados inadecuados o de baja calidad empeoran la experiencia de las mujeres, lo que interfiere en el trabajo de parto, prolongándolo y desencadenando lo que se conoce como "cascada del intervencionismo" (OMS 1996 19), donde el último escalafón es la cesárea. Cabe preguntarse, por tanto, ¿qué cuidados inadecuados están afectando la experiencia de parto de las mujeres en Nicaragua, perjudicando la fisiología del parto y presionando al alza las tasas de cesáreas?

Por su parte, en el año 2014, la OMS emitió una declaración donde se reconocía que la situación en relación con el ejercicio de los derechos humanos (DDHH) de las mujeres durante el parto era alarmante y donde se instaba a investigar en profundidad la violencia obstétrica, cómo prevenirla y cómo erradicarla.

En particular, los $\mathrm{DSR}^{5}$ son sistemáticamente vulnerados durante el parto a través de distintas formas de violencia (institucional, verbal, física o simbólica), ya que dentro del modelo actual de atención al parto institucional (hegemónico en la mayor parte de los países del mundo), el ejercicio de estos derechos durante el parto se revela 
imposible para las mujeres. "Dado que el placer es un aspecto intrínseco de la sexualidad, el derecho a buscarlo, expresarlo y a determinar cuándo experimentarlo no debe ser negado a persona alguna" (Sharpe principio n. ${ }^{\circ}$ 4). A través de la autonomía individual y relacional, el placer se hace posible también durante el parto, un espacio para la expresión de la sexualidad no falocéntrica ni heterosexista (Massó 522). Sin embargo, dentro del modelo actual, la imposición de protocolos hospitalarios a las mujeres durante sus partos, imposibilita el ejercicio efectivo de esta autonomía, por lo que en la práctica se impide el ejercicio del derecho al placer.

La sobretasa de cesáreas también está vinculada con la violencia contra las mujeres durante el parto: más allá de las cesáreas innecesarias que son llevadas a cabo por incentivos económicos (Arrieta y Oneto 27, 32) y que son una expresión clara de violencia obstétrica, hay cesáreas de urgencia que terminan siendo necesarias a consecuencia de otras expresiones de la violencia obstétrica, como la administración inadecuada de oxitocina sintética, la percepción de encontrarse en un entorno inseguro, la no facilitación de información oportuna para el autocuidado durante el parto, la privación de apoyo emocional durante todo el parto o en alguna de sus fases o la misma construcción social del parto como un evento de riesgo.

Las distintas manifestaciones de violencia obstétrica interfieren de forma negativa en la salud del parto (Olza 5; Fernández 114-117; Odent 2004 27) afectando la segregación de oxitocina endógena, hormona que se produce principalmente en el hipotálamo y que es crucial para el desarrollo de las contracciones uterinas que posibilitan un parto saludable (Alba et al. 27-28; Olza 5). Por tanto, la actual sobretasa de cesáreas en el sistema de salud debe ser estudiada también en relación a la violencia obstétrica que experimentan las mujeres en el marco de la atención al parto realizado dentro de la institución hospitalaria (Hodges 9).

\section{Metodología}

Nicaragua no cuenta con estudios donde se vinculen la tasa de cesáreas y la calidad de la atención al parto desde la óptica de las experiencias de las mujeres que han vivido un parto. Es posible encontrar estudios realizados a partir de las historias clínicas y de las percepciones de profesionales y gestores del sector salud (Colomar et al. 2384; Narváez 23). Sin embargo, estos trabajos no ofrecen información sobre prácticas inadecuadas que podrían estar presionando al alza la tasa de cesáreas y que parecen estar presentes de forma habitual en la atención al parto institucional. Así lo sugieren entrevistas informales que he mantenido con mujeres con experiencias de parto, con obstetras y con enfermeras. Frecuentemente, las mujeres con experiencias de parto señalan en estas entrevistas haber sufrido tactos traumáticos, procedimientos no consentidos (como la maniobra de Kristeller o la maniobra de Hamilton) ${ }^{6}$ y desprecio hacia ellas o sus acompañantes. 
Con la finalidad de revelar la presencia de estas y otras prácticas inadecuadas en la atención al parto, el diseño de esta investigación de tipo exploratorio toma las experiencias de parto y de cesárea de las mujeres como objeto de estudio. El diseño metodológico es de tipo mixto, recopilando datos de tipo cuantitativo y cualitativo. En correspondencia con su naturaleza exploratoria, el tamaño muestral no ofrece resultados estadísticamente significativos a nivel poblacional.

\section{Objetivos general y específicos}

El objetivo general de este estudio es explorar la calidad de los cuidados facilitados durante el parto y la cesárea a mujeres residentes en las áreas urbanas de la costa pacífica de Nicaragua. Al respecto, considero el nacimiento puede tener lugar por dos vías distintas:

Parto: Evento fisiológico donde la mujer pare un bebé, este es desplazado debido a la acción de contracciones desde el útero hacia el exterior, a través del cuello uterino y del canal vaginal. Si la mujer adopta una postura fisiológica cuando está pariendo, la gravedad también facilita el desplazamiento del bebé hacia el exterior. El parto es, por tanto, un evento propio de las mujeres y, per se, solo puede ser vaginal.

Cesárea (urgente o programada): El nacimiento se produce a través del abdomen, mediante una cirugía mayor donde se realiza una incisión sobre la pared abdominal y sobre el útero. específicos:

Para lograr el objetivo general del estudio, se plantearon cuatro objetivos

Obtener una valoración de los cuidados al parto de acuerdo con las experiencias de parto o cesárea de mujeres residentes en las áreas urbanas de la costa pacífica de Nicaragua.

- $\quad$ Estudiar la tasa de cesáreas de la muestra en relación con la tasa ideal de cesáreas, tomando esta tasa como un indicador de resultado y de calidad de los cuidados recibidos durante el parto.

- Verificar en la muestra estudiada la aplicación de las recomendaciones de la OMS para los cuidados al parto.

- Valorar el cumplimiento del derecho al consentimiento informado de las mujeres participantes en relación a los cuidados recibidos durante el parto.

\section{Mujeres participantes en el estudio}

Los datos analizados fueron obtenidos a través de un cuestionario aplicado en mayo del 2013 a 24 mujeres que habían tenido una experiencia de parto o cesárea entre el 2008 y el 2013. El 63 por ciento de los nacimientos tuvieron lugar en Managua 
y el 29 por ciento en otras cabeceras departamentales. El 8 por ciento de las mujeres no ofreció este dato. Todos los nacimientos se produjeron en instituciones hospitalarias.

En relación a su caracterización socioeconómica, dieciocho de las participantes eran profesionales, tres ejercían actividades no cualificadas, 1 era estudiante y una mujer no ofreció este dato. Veinte mujeres contaban con una edad comprendida entre 20 y 30 años en el momento del nacimiento del bebé y cuatro presentaban entre 31 y 35 años. El 79 por ciento (19 mujeres) no habían parido ni se había sometido a una cesárea con anterioridad.

Figura 4

CARACTERIZACIÓN DE LA MUESTRA

Ocupación principal

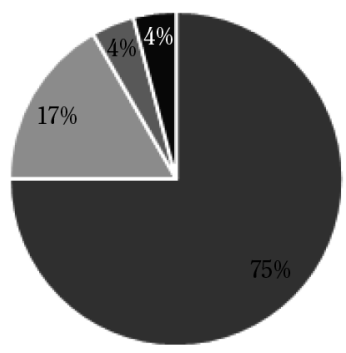

Edad durante el nacimiento

- Profesionales

-Otras ocupaciones

- Estudiantes

- Sin dato

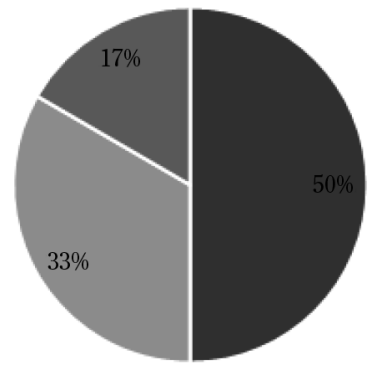

- De 20 a 25 años

- De 26 a 30 años

- De 31 a 35 años

Fuente: Elaboración propia a partir de datos del estudio.

Las participantes fueron identificadas a partir de la técnica de bola de nieve. El tamaño muestral quedó definido por el número de mujeres que decidieron participar en el estudio, cerrando la fase de recogida de datos dos meses después de su inicio. En total participaron 32 mujeres, que tras la depuración de la base de datos, determinó un tamaño muestral de 24 casos.

Para que las mujeres pudiesen ejercer su derecho al consentimiento informado, en el instrumento de recogida de información se indicó que la colaboración en el estudio era completamente voluntaria y se explicó su finalidad, proporcionando medios de contacto adicionales para obtener más información al respecto, si fuese necesario. El compromiso con el tratamiento confidencial de los datos fue establecido expresamente a través de este mismo instrumento. 


\section{Instrumento de recolección de información}

Como instrumento de recogida de información se elaboró un cuestionario con dieciocho ítems para describir los cuidados recibidos, más siete ítems para caracterizar la muestra y obtener variables de control. De los dieciocho ítems, quince de ellos eran preguntas cerradas tipo test para recabar datos sobre el seguimiento de las recomendaciones de la OMS para los cuidados al parto. Se integró una pregunta semiabierta para describir la posición usada durante el expulsivo, y dos preguntas abiertas para valorar la experiencia de parto o cesárea, solicitando a las participantes una relación de los aspectos que les hubiese gustado cambiar en los cuidados recibidos. De acuerdo con este cuestionario se construyeron 23 variables cuantitativas (21 nominales y 2 ordinales).

Las participantes entregaron el cuestionario por escrito. Con la finalidad de velar por la adecuada compresión del cuestionario, este instrumento fue sometido a la revisión de dos personas no especializadas en cuidados al parto.

\section{Análisis de los datos}

\section{Análisis de la aplicación de las recomendaciones para la atención al parto}

El análisis de la aplicación de las recomendaciones de la OMS para los cuidados al parto se realizó comparando los cuidados recibidos por las participantes en el estudio contra una selección de cuidados orientados por la OMS. Este análisis se realizó a través de 11 indicadores construidos al efecto, comparando el valor observado en la muestra contra el estándar recomendado. Los indicadores seleccionados para efectuar este análisis comparativo fueron los siguientes:

1. Tasa de cesáreas global, distinguiendo por tipo de cesárea (urgente o programada) y por la condición de nacimientos previos.

2. Porcentaje de mujeres con experiencia de parto o nacimiento por cesárea urgente con libertad de movimiento durante la dilatación.

3. Porcentaje de mujeres con experiencia de parto que no parieron en decúbito supino. ${ }^{7}$

4. Porcentaje de mujeres con experiencia de parto que ingirieron líquidos durante la dilatación.

5. Porcentaje de mujeres con experiencia de parto a las que se le aplicó un enema.

6. Porcentaje de mujeres con experiencia de parto a las que se le realizó una episiotomía.

7. Porcentaje de mujeres que sufrieron maniobra de Kristeller, distinguiendo por tipo de nacimiento (parto o cesárea urgente).

8. Porcentaje de mujeres que practicaron el apego precoz. 
9. Porcentaje de mujeres acompañadas por un familiar u otro acompañante elegido.

10. Porcentaje de mujeres que se sintieron cómodas y tratadas con calidez durante el parto.

11. Porcentaje de mujeres que declararon haber ejercido el consentimiento informado de los cuidados recibidos.

La selección de los cuidados a analizar se realizó tomando como referencia las orientaciones emitidas por la OMS a través de la "Declaración de Fortaleza sobre las Recomendaciones de la OMS sobre el nacimiento" (1985), y a través del documento "Cuidados en el parto normal: una guía práctica" (1996).

Cuando estos documentos no abordaron con claridad suficiente un cuidado o práctica, o no establecieron un estándar para el indicador, se recurrió a las recomendaciones de la OMS para la conducción del trabajo de parto (2015) y a las Normativas 042, 077, 109, 030 y 111 del Ministerio de Salud de Nicaragua (2008, 2010a, 2010b, 2011, 2013). Parte de los cuidados analizados son propios de las competencias del personal de salud y otros deben ser facilitados por todas las personas cuidadoras presentes en el nacimiento, ya sea familiar o profesional de la salud.

Hay indicadores, como es el caso de la tasa de episiotomías, cuyo cálculo es pertinente solo si se estableció trabajo de parto. En estos casos, he asumido que en todas las cesáreas urgentes siempre se estableció trabajo de parto.

\section{Análisis de las experiencias de las mujeres en la atención al parto}

Los datos recogidos en relación a la vivencia del parto fueron analizados aplicando la técnica del análisis del contenido, identificando 5 categorías y 27 temas relacionados con la calidad de los cuidados al parto (Tabla 1). El análisis de los resultados se realizó clasificando la información en dos secciones:

Prácticas que dañan: prácticas que fueron realizadas y que fueron valoradas como negativas o lesivas por las mujeres participantes.

Prácticas que cuidan: cuidados valorados como positivos por las mujeres durante el parto, algunos de los cuales se llevaron a cabo y otros no. 
Tabla 1

CATEGORÍAS Y TEMAS IDENTIFICADOS A TRAVÉS DE LA TÉCNICA DEL ANÁLISIS DEL CONTENIDO

\begin{tabular}{|c|c|}
\hline Calidad cuidados & $\begin{array}{l}\text { Déficit de cuidados x profesionales- abandono } \\
\text { Inadecuados por no incorporar información aportada por la mujer } \\
\text { Mix de cuidados en las cesáreas- cuello de botella } \\
\text { Trato deshumanizado } \\
\text { Tactos inadecuados (demasiado frecuentes/varias personas) } \\
\text { Separación madre-bebé } \\
\text { Importancia del apoyo afectivo } \\
\text { No libertad de movimientos } \\
\text { Conducción } \\
\text { Inducción } \\
\text { Enema } \\
\text { No uniformidad en el proceso de atención } \\
\text { Maniobra kristeller }\end{array}$ \\
\hline Condiciones para los cuidados & $\begin{array}{l}\text { Medios mejorables } \\
\text { RRHH escasos }\end{array}$ \\
\hline Consentimiento informado & $\begin{array}{l}\text { Falta del deber de informar } \\
\text { No participación de la mujer en las decisiones sobre los cuidados } \\
\text { No respeto a la decisión de la mujer } \\
\text { Falta del deber de informar adecuadamente o negligencia }\end{array}$ \\
\hline Impactos a largo plazo & Miedo al parto \\
\hline Valoración global de los cuidados & $\begin{array}{l}\text { Muy negativa/traumática } \\
\text { Muy positiva } \\
\text { Negativa } \\
\text { No satisfactoria } \\
\text { No uniforme } \\
\text { Positiva } \\
\text { Positiva con matices }\end{array}$ \\
\hline
\end{tabular}

Fuente: Elaboración propia a partir de datos del estudio.

\section{Evaluación de la aplicación de las recomendaciones para los cuidados al parto: Análisis de los indicadores de atención al parto}

\section{Vía del nacimiento}

El análisis de los resultados cuantitativos inicia con el examen de la tasa de cesáreas en la muestra estudiada, dado su uso como indicador de resultado y de calidad de los cuidados al parto. La tasa global de cesáreas de la muestra alcanzó 
el 63 por ciento, muy alejado del umbral máximo de la tasa ideal de cesáreas recomendada por la OMS (Tabla 2), establecido entre el 10 por ciento y el 15 por ciento (OM Declaración 3-4). Esta tasa en la muestra estudiada es similar a la tasa promedio registrada para el régimen de salud de financiación privada en el periodo 2006-2012 (69 por ciento) (INIDE 2013), así como para el régimen contributivo en el 2016 (58 por ciento) (Instituto Nacional de la Seguridad Social (INSS)).

Tabla 2

TASA DE CESÁREA MUESTRAL

\begin{tabular}{|c|c|c|c|c|c|c|}
\hline Indicador & \multicolumn{2}{|c|}{ Subindicadores } & Estándar & \multicolumn{3}{|c|}{ Resultado muestral } \\
\hline \multirow{4}{*}{$\begin{array}{l}\text { Tasa de } \\
\text { cesáreas global } \\
\text { ( } 24 \text { partos) }\end{array}$} & \multirow{2}{*}{$\begin{array}{c}\text { Primíparas } \\
\text { (19 partos) }\end{array}$} & Cesáreas urgentes & \multirow{4}{*}{$\begin{array}{c}5 \% \leq \text { Tasa ideal } \\
\text { de cesáreas } \\
\leq 15 \%\end{array}$} & \multirow{4}{*}{$63 \%$} & \multirow{2}{*}{$63 \%$} & $47 \%$ \\
\hline & & Cesáreas programadas & & & & $16 \%$ \\
\hline & \multirow{2}{*}{$\begin{array}{c}\text { Multíparas } \\
\text { (5 partos) }\end{array}$} & Cesáreas urgentes & & & \multirow{2}{*}{$60 \%$} & $20 \%$ \\
\hline & & Cesáreas programadas & & & & $40 \%$ \\
\hline
\end{tabular}

Fuente: Elaboración propia a partir de datos del estudio. Nota: De las 24 participantes, 9 tuvieron experiencias de parto y 13 tuvieron cesáreas.

En las mujeres primíparas (sin partos anteriores), la tasa de cesáreas es también del 63 por ciento. Debido a la pervivencia en la práctica obstétrica del mito "una vez cesárea, siempre cesárea", este dato es especialmente alarmante, ya que en la práctica supone una indicación absoluta de cesárea para las mujeres con una cesárea previa. Esta máxima fue enunciada en 1916 por Edwin Cragin para advertir sobre la necesidad de evaluar concienzudamente la necesidad de una primera cesárea, dadas las limitaciones de la ciencia médica de la época -sin acceso a antibióticos ni a transfusiones y con cuidados anestésicos e higiénicos muy deficientes- y dados los riesgos mayores que suponía la técnica quirúrgica utilizada en aquel entonces, que era la de la cesárea clásica o vertical (Cunningham 609). Muy pocas cesáreas se hacen en la actualidad usando esta técnica, indicada solamente en situaciones muy específicas. Es evidente además que los recursos y las técnicas de la medicina han evolucionado increíblemente en el último siglo, por lo que la "advertencia" de Edwin Cragin ha dejado de tener sentido.

Sin embargo, en Nicaragua se ha instalado como un mito en la práctica obstétrica, y cada año miles de mujeres siguen siendo innecesariamente sometidas a cesárea. Muestra de ello es el alto porcentaje de mujeres con cesáreas programadas entre las multíparas: posiblemente, gran parte de estas mujeres tengan una cesárea previa. Así consta también en las conclusiones del estudio llevado a cabo por Alexis Narváez, según el cual la cesárea previa fue la indicación más frecuente para realizar cesárea 
en el Hospital Bertha Calderón ${ }^{8}$ en el 2014, alcanzando el 35 por ciento de las cesáreas totales (29) y el 16 por ciento de los nacimientos totales.

En contraste, organismos rectores de la práctica obstétrica en distintos países, ${ }^{9}$ concuerdan en que el Parto Vaginal Después de Cesárea (PVDC) planificado es una opción segura para la mayoría de las mujeres con un historial de cesárea en el segmento inferior del útero. ${ }^{10}$

En relación a la oxitocina sintética, tanto los datos recogidos por esta muestra, como el estudio de Alexis Narváez nos indican que su uso es habitual durante el trabajo de parto en Nicaragua: el 67 por ciento de las mujeres participantes con partos fueron medicadas con oxitocina sintética, mientras que en el estudio de Narváez el porcentaje sube hasta el 94 por ciento (29). Además, al 64 por ciento de las mujeres participantes con partos o con cesárea urgente indicaron que los profesionales de salud les rompieron las membranas de forma artificial. La práctica rutinaria de la administración de oxitocina sintética y de la rotura artificial de membranas amnióticas están contraindicadas como medida de prevención del retraso en el trabajo de parto (OMS 2015 17, 20). ${ }^{11}$ El cuestionario aplicado en este estudio no facilitaba elementos suficientes para valorar si la aplicación de estos procedimientos fue indicada de forma correcta o si se realizaron de manera rutinaria, por lo que no se pueden expresar conclusiones al respecto. Sin embargo, tanto los datos recogidos en esta muestra como los recogidos a través del estudio de Narváez, ponen en cuestión la justificación de estas prácticas.

Sumado a lo anterior, es necesario señalar que el 35 por ciento de las participantes con partos y nacimientos por cesárea urgente no sabían si les habían administrado oxitocina sintética, lo que sugiere la posibilidad de una violación de los derechos a la salud de estas mujeres, al no ser correctamente informadas sobre las prácticas realizadas.

\section{Libertad de movimiento e ingesta de líquidos en la dilatación. Libertad de posición en el expulsivo}

En sus recomendaciones para los cuidados al parto normal, la OMS indica que se debe promover y permitir la movilidad de la mujer y la ingesta de líquidos durante la fase de dilatación. También indica que la posición de litotomía (tumbada boca arriba) es la peor para parir por ser la posición que más dificulta el expulsivo $(1996$ 37, 17, 50).

Por el contrario, en la Tabla 3 se observa como ninguna de estas recomendaciones se aplicó de forma satisfactoria en los partos de estas mujeres, siendo el indicador referido a la posición durante el expulsivo, el que presenta el valor más bajo: solo una de cada cuatro mujeres parió en un postura propicia para la fisiología del parto. Además, al 40 por ciento de las mujeres se les restringió el movimiento, y solo el 40 por ciento pudo beber líquidos durante el periodo de dilatación. 
Tabla 3

LIBERTAD DE MOVIMIENTO E INGESTA DE LÍQUIDOS EN LA DILATACIÓN. LIBERTAD DE POSICIÓN EN EL EXPULSIVO

\begin{tabular}{lcc}
\hline Indicador & Estándar & $\begin{array}{c}\text { Resultado } \\
\text { muestral }\end{array}$ \\
\hline $\begin{array}{l}\text { Porcentaje de mujeres con parto por vía vaginal o cesárea urgente con } \\
\text { libertad de movimiento durante la dilatación }\end{array}$ & $100 \%$ & $58 \%$ \\
\hline $\begin{array}{l}\text { Porcentaje de mujeres con parto por vía vaginal que no parieron } \\
\text { en posición de litotomía }\end{array}$ & $100 \%$ & $25 \%$ \\
\hline $\begin{array}{l}\text { Porcentaje de mujeres con parto por vía vaginal que ingirieron líquidos } \\
\text { durante la dilatación }\end{array}$ & $100 \%$ & $38 \%$ \\
\hline
\end{tabular}

Fuente: Elaboración propia a partir de datos del estudio.

\section{Enema, episiotomía y maniobra de Kristeller}

El análisis de estos indicadores pone de manifiesto la persistencia de prácticas en la atención al parto sin evidencia científica que las avale, generando perjuicios y riesgos para la mujer o la criatura recién nacida.

Tabla 4

ENEMA, EPISIOTOMÍA Y MANIOBRA DE KRISTELLER

\begin{tabular}{|c|c|c|c|c|}
\hline Indicadores & Subindicadores & Estándar & \multicolumn{2}{|c|}{$\begin{array}{l}\text { Resultado } \\
\text { muestral }\end{array}$} \\
\hline \multicolumn{2}{|c|}{$\begin{array}{l}\text { Porcentaje de mujeres con parto por vía vaginal a las que se } \\
\text { le aplicó un enema }\end{array}$} & $0 \%$ & \multicolumn{2}{|c|}{$20 \%$} \\
\hline \multicolumn{2}{|c|}{$\begin{array}{l}\text { Porcentaje de mujeres con parto por vía vaginal a las que se } \\
\text { le realizó una episiotomía }\end{array}$} & $\begin{array}{c}8 \% \leq \text { Tasa } \\
\text { episiotomías } \\
\text { selectivas } \leq 30 \%\end{array}$ & \multicolumn{2}{|c|}{$88 \%$} \\
\hline \multirow{2}{*}{$\begin{array}{l}\text { Porcentaje de mujeres con parto por } \\
\text { vía vaginal o cesárea urgente con } \\
\text { maniobra de Kristeller }\end{array}$} & Partos por vía vaginal & \multirow[b]{2}{*}{$0 \%$} & \multirow[b]{2}{*}{$33 \%$} & $44 \%$ \\
\hline & $\begin{array}{l}\text { Partos por cesárea } \\
\text { urgente }\end{array}$ & & & $22 \%$ \\
\hline
\end{tabular}

Fuente: Elaboración propia a partir de datos del estudio.

Ya en 1996, la OMS indicaba que la aplicación rutinaria de enema era claramente perjudicial o ineficaz (68). Sin embargo, se le aplicó este procedimiento a una de cada 
cinco mujeres con experiencia de parto. Resta saber, cuántas de ellas estuvieron conformes con su aplicación.

En relación a la episiotomía, esta es una práctica de cirugía menor en la que se realiza una incisión sobre el perineo de la mujer (entre la vagina y el ano) durante el trabajo de parto, con la finalidad de agrandar el canal vaginal. La episiotomía incrementa la morbilidad materna, aumentando el riesgo de sufrir desgarros en el suelo pélvico de III y IV grado (Landy et al.; Hartmann et al.). Esta cirugía fue realizada a 9 de cada 10 mujeres con experiencias de parto, lo que evidencia que se trata de una práctica rutinaria por parte del personal del sector salud. Ello contraviene la evidencia científica, que orienta la práctica de la episiotomía de forma selectiva, ${ }^{12}$ fijando el estándar entre el 10 por ciento y el 20 por ciento de los partos (OMS 1996 55; Wagner 7), muy alejado del valor registrado en esta muestra.

Por último, vemos también una brecha importante entre el estándar para el indicador de la maniobra de Kristeller, cuya práctica no está respaldada por la evidencia científica, y el valor que este indicador alcanzó en la muestra estudiada: el 33 por ciento de las mujeres con trabajo de parto sufrió esta práctica, de este porcentaje el 44 por ciento para las mujeres con experiencias de parto y del 22 por ciento para las mujeres con cesárea urgente. Esta maniobra ha sido prohibida legalmente en el Reino Unido, Chile y Alemania, debido a los graves riesgos que supone para la salud de la madre (entre ellos rotura uterina, hemorragia y fractura costal) y de la criatura recién nacida (lesiones de órganos internos, hipoxia y parálisis de Erb, entre otros) (Campos 23; Asociación El Parto es Nuestro 1).

\section{Apego precoz, acompañamiento y calidez}

El término apego precoz no cuenta con una definición consensuada a nivel interinstitucional, siendo tal vez la más aceptada la definición adoptada por la Iniciativa para la Humanización de la Asistencia al Nacimiento y la Lactancia (iHan). Este programa de la UNICEF y la OMS lo define como un cuidado a la salud en el que se coloca a la criatura recién nacida en contacto directo piel con piel con su madre, inmediatamente después del parto y de forma ininterrumpida por lo menos durante una hora (iHan 2016 4). Otros organismos señalan un contacto mínimo de dos horas (MINSA Normativa 108 40).

Además de ser una práctica que cuida (es decir, un cuidado) altamente recomendada por la OMS (1996 23) y con claras evidencias científicas a su favor, el apego precoz es un derecho de las niñas y los niños reconocido en el artículo 9.1 de la Convención de las Naciones Unidas sobre los Derechos del Niño (CDN), según el cual las criaturas recién nacidas no deben ser separadas de sus madres contra la voluntad de estas. ${ }^{13}$

A pesar de ello, en la muestra estudiada solo la mitad de las mujeres recibieron este cuidado durante el parto (Tabla 5). Como era de esperar, los porcentajes más 
altos de apego precoz se dieron en las experiencias de parto, no encontrándose ningún caso de cesárea programada con práctica de apego precoz.

Según los datos muestrales, el 50 por ciento de las mujeres con experiencias de parto por cesárea urgente declararon haber tenido contacto piel con piel con su bebé. Sin embargo, entrevistas informales mantenidas con mujeres con experiencias de cesárea urgente que no participaron en este estudio, sugieren la posibilidad de que este valor no esté reflejando la práctica habitual durante este procedimiento quirúrgico. Es posible que la imprecisión del término "piel con piel" y la falta de una definición más detallada dentro del cuestionario hayan conducido a sobredimensionar este subindicador.

Tabla 5

APEGO PRECOZ, ACOMPAÑAMIENTO Y CALIDEZ

\begin{tabular}{|c|c|c|c|c|}
\hline Indicador & Subindicadores & Estándar & \multicolumn{2}{|c|}{ Resultado muestral } \\
\hline \multirow{3}{*}{$\begin{array}{l}\text { Porcentaje de mujeres que practicaron el } \\
\text { apego precoz }\end{array}$} & Vía vaginal & \multirow{3}{*}{$100 \%$} & \multirow{3}{*}{$50 \%$} & $78 \%$ \\
\hline & Cesárea urgente & & & $50 \%$ \\
\hline & Cesárea programada & & & $0 \%$ \\
\hline \multirow{3}{*}{$\begin{array}{l}\text { Porcentaje de mujeres acompañadas por } \\
\text { un familiar u otro acompañante elegido }\end{array}$} & Vía vaginal & \multirow{3}{*}{$100 \%$} & \multirow{3}{*}{$78 \%$} & $86 \%$ \\
\hline & Cesárea urgente & & & $75 \%$ \\
\hline & Cesárea programada & & & $67 \%$ \\
\hline \multicolumn{2}{|c|}{$\begin{array}{l}\text { Porcentaje de Mujeres que se sintieron cómodas y tratadas con } \\
\text { calidez durante el parto }\end{array}$} & $100 \%$ & \multicolumn{2}{|c|}{$100 \%$} \\
\hline
\end{tabular}

Fuente: Elaboración propia a partir de datos del estudio.

El acompañamiento de personas integradas en las redes familiares o comunitarias de la mujer es otra de las recomendaciones de la OMS (1996 23). Entre las mujeres de la muestra que solicitaron apoyo familiar o social durante el parto o la cesárea, una de cada cuatro no pudo ejercer el derecho a recibir este tipo de cuidados no profesionales. Nuevamente, se observa que en los nacimientos por cesárea la implementación de las recomendaciones estudiadas es menos frecuente. Así mismo, destaca que el 25 por ciento de las mujeres no solicitase el apoyo de acompañantes no profesionales. Este estudio no recogió datos suficientes para explicar este hallazgo.

Desde un punto de vista más comprehensivo, esta recomendación se erige además como uno de los pilares de la atención al parto, puesto que el parto forma parte de la sexualidad de las mujeres, siendo una parte de su vida privada y familiar, por lo que es su derecho decidir sobre las prácticas y conductas sexuales que desean realizar 
durante el mismo, tanto a nivel individual como relacional. Esto quiere decir que las mujeres tienen derecho a decidir qué personas quieren que participen en sus partos. El derecho a la libertad sexual abarca tanto la capacidad de decidir cómo vivir nuestra sexualidad, como con qué personas experimentarla (Federación Internacional de Planificación de la Familia art 4, 5, 7).

Desde los aportes de la ética de los cuidados a la ética biomédica, el principio de la autonomía se enfoca desde una perspectiva más amplia, en la que los vínculos relacionales son de especial importancia para la vivencia del parto: la concepción liberal del sujeto independiente, de la que emana el concepto de autonomía procedente del mundo anglosajón, no facilita la implementación de medidas que permitan establecer una relación satisfactoria entre el personal de salud y las mujeres que están pariendo, pues cuando estas son aisladas de su contexto familiar y social dentro de la institución hospitalaria, son privadas de los vínculos relacionales que precisamente les confieren la autonomía necesaria para transitar con confianza por este periodo vital en el que la vulnerabilidad -cualidad intrínseca a la naturaleza humana- se hace especialmente presente (Delgado 2012 29-32, 2017 98; Feito 2011 1, 5-6). El entendimiento de la atención al parto desde el enfoque de la autonomía relacional, permite comprender mejor como la privación o limitación del apoyo familiar y social durante el parto, acrecienta la vulnerabilidad de las mujeres dentro del entorno hospitalario, pues "lo que realmente hace posible la autonomía no es la separación de los demás, sino más bien las relaciones, los vínculos" (Delgado 2012 28).

Además de las dimensiones materiales y morales del cuidado, también su dimensión afectiva o emocional nos impela a integrar el apoyo familiar y comunitario como un pilar dentro del modelo de atención al parto. La violencia que de forma implícita emana de la negación de los aspectos afectivos y emocionales de los cuidados a la salud normativizados desde el modelo biomédico de salud y el paradigma tecnocrático de atención al parto (Poblete y Valenzuela 2007 501; Cabral 2002 79-80, 2008 581), ha sido uno de los aspectos que ha motivado el nacimiento de distintos movimientos ciudadanos por la humanización de la atención al parto a nivel mundial. En el ámbito de las políticas públicas, y desde un enfoque más global, el modelo del cuidado social o social care ha surgido como una propuesta alternativa al actual esquema organizativo de los cuidados. Este modelo, desarrollado en sus orígenes por Mary Daly y Jane Lewis, entiende los cuidados desde una perspectiva transversal, no fragmentada y multidimensional, y considera que la responsabilidad de su provisión es tanto una responsabilidad colectiva de la sociedad como una responsabilidad moral de las personas, por lo que su sostenimiento requiere integrar todas las actividades y relaciones implicadas en el sostenimiento de las necesidades físicas y emocionales de las personas (Palomo 343, 344).

Por último, el apoyo afectivo y un ambiente cálido y agradable fomentan el bienestar físico y emocional de la mujer y mejoran la experiencia del parto (OMS 1996 67, 22). 
En sintonía con los hallazgos de Michelle Sadler (2004 21-24), este estudio revela que a pesar de los déficits en el seguimiento de las recomendaciones para la atención al parto, todas las mujeres de la muestra declararon haberse sentido cómodas y tratadas con calidez durante el parto.

\section{Consentimiento informado}

Con la finalidad de crear las condiciones que permitan el ejercicio del principio de autonomía tal y como es entendido desde la ética biomédica angloamericana, se ha promovido la implementación del consentimiento informado en la relación establecida entre pacientes y personal médico. De acuerdo con la regulación de esta práctica en los artículos 8.4 y 8.8 de la Ley General de Salud (Asamblea Nacional de la República de Nicaragua 2002), en este estudio se entiende que las mujeres ejercieron su derecho al consentimiento informado cuando el personal de salud facilitó la información necesaria para comprender los riesgos e indicaciones de los tratamientos recomendados y sobre sus alternativas, y cuando su consentimiento para aplicar dichos tratamientos fue solicitado expresamente.

Los datos indican que solo un 11 por ciento de las mujeres de la muestra declararon que los cuidados y procedimientos médicos realizados fueron aplicados tras cumplir ambos requisitos (Tabla 6). Como se observa en la Tabla 6, el 58 por ciento de las mujeres indicó que se le informó sobre los cuidados y procedimientos médicos indicados, y el 68 por ciento expresó que el personal de salud solicitó su consentimiento, pero solo el 11 por ciento declaró que se habían producido ambas circunstancias al mismo tiempo, condición necesaria para entender la realización efectiva del consentimiento informado.

Tabla 6

CONSENTIMIENTO INFORMADO

\begin{tabular}{|c|c|c|c|c|}
\hline Indicador & Subindicadores & Estándar & & \\
\hline \multirow{2}{*}{$\begin{array}{l}\text { Porcentaje de mujeres que } \\
\text { declararon haber recibido los } \\
\text { cuidados al parto bajo el con- } \\
\text { sentimiento informado }\end{array}$} & $\begin{array}{l}\text { Porcentaje de mujeres que } \\
\text { declararon haber sido } \\
\text { informadas sobre los } \\
\text { cuidados recibidos }\end{array}$ & \multirow[t]{2}{*}{$100 \%$} & \multirow[t]{2}{*}{$11 \%$} & $58 \%$ \\
\hline & $\begin{array}{l}\text { Porcentaje de mujeres que } \\
\text { declararon que su } \\
\text { consentimiento fue solicitado }\end{array}$ & & & $68 \%$ \\
\hline
\end{tabular}

Fuente: Elaboración propia a partir de datos del estudio. 


\section{Resultados cualitativos: La valoración de las mujeres sobre sus experiencias de parto}

\section{Prácticas que dañan}

Dentro de las prácticas que dañan, las participantes pusieron el acento sobre todo en dos prácticas: los tactos vaginales y la maniobra de Kristeller.

Los tactos vaginales se realizan para determinar el inicio y el avance de la dilatación. La OMS pauta su realización cada cuatro horas señalando que en cualquier caso "se deben priorizar los deseos y preferencias de las mujeres reduciendo al mínimo el número total de tactos vaginales" (2015 14), pues es un procedimiento invasivo y molesto que además puede causar dolor. Dentro del marco normativo del MINSA no se encontraron orientaciones normativas al número de tactos vaginales.

Dos de las participantes se refirieron a los tactos vaginales como una práctica que habían rechazado, pero que no pudieron impedir. Una de estas mujeres (Caso 13) señaló que los tactos vaginales realizados por el personal de salud habían sido traumáticos: "Ante todo [la experiencia del nacimiento fue] dolorosa e incómoda, lo más traumático para mí fue la revisión para la dilatación que fue cada dos horas y la raquídea para la cesaría" (Caso 13). En el Caso 2, la mujer se refiere a la práctica de tactos vaginales por parte de varios miembros del personal médico, lo que contraría las buenas prácticas para la atención al parto: los tactos vaginales deben ser realizados por una sola persona ya que su utilidad para medir el avance de la dilatación está limitada por la subjetividad propia del procedimiento (FAME 1). En este caso, la mujer usó comillas para reproducir los motivos aducidos por los profesionales que la atendieron: “Durante el proceso de labor hubo intervención de varios médicos, cada uno hacia tacto para "llevar control de dilatación"”" (Caso 2).

La maniobra de Kristeller ${ }^{14}$ también fue señalada como una de las maniobras más traumáticas para las mujeres. Así lo indica una de las participantes que, dentro de la experiencia de un parto inducido, rechazó sobre todo la maniobra de Kristeller: “a pesar de que fue parto inducido, me hubiese gustado cambiar algunas cosas, principalmente la maniobra Kristeller" (Caso 20).

Una de las mujeres indicó que el trato recibido durante la fase del expulsivo había sido deshumanizante: “En el proceso de dilatación me trataron con amabilidad y respeto, pero en la sala de partos fui totalmente ignorada por doctores y enfermeras. La oxitocina y la episiotomía eran innecesarias. Tampoco me gustó que en la sala de partos fuera tratada como el objeto que trae el producto" (Caso 1).

Por su parte, una de las mujeres describió como traumática su experiencia en el marco de una cesárea programada con cesárea anterior, sin llegar a explicar el motivo: "la primera no fue así, está fue muy traumática" (Caso 22). 
También se recogió un caso de abandono por parte del personal médico y de enfermería, incumpliendo su deber de cuidar a la mujer durante el parto: "las primeras 6 horas que me mandaron sola a caminar fueron dolorosas, de intranquilidad y angustia pues no había nadie diciéndome que hacer, explicándonos lo que pasaba" (Caso 8).

\section{Prácticas que cuidan}

Entre las prácticas que cuidan, el apego precoz fue considerado por las mujeres como uno de los cuidados más relevantes para vivir una experiencia de nacimiento satisfactoria: "me hubiese gustado que dejaran al bebe conmigo luego de la operación" (Caso 11), “Me hubiese gustado... [el] contacto con el bebé en cuanto salga" (Caso 1), "tardaron demasiado en enseñarme al bebé" (Caso 6).

Cuatro de las participantes (el 17 por ciento de la muestra) destacaron la importancia del apoyo emocional, tanto por parte del personal de salud como por parte de las familias: "este proceso es siempre doloroso y estresante, pero con el apoyo recibido tanto del médico que te atiende como de la familia en especial del esposo se hace menos tenso" (Caso 13), “Considero importante la compañía de la pareja o algún familiar durante el parto, decisión que debería ser respetada" (Caso 21).

\section{En relación a la importancia del consentimiento informado}

Finalmente, las participantes en este estudio indicaron que la autonomía sobre su propio cuerpo, el derecho a vivir y decidir sobre su parto y el derecho a ser informadas sobre su salud, eran esenciales para vivir el nacimiento de un bebé como una experiencia de vida positiva, siendo la vulneración de estos derechos una fuente importante de ansiedad durante el parto.

Más allá del indicador sobre el ejercicio del consentimiento informado, el cual como vimos era muy bajo, a través de las preguntas abiertas las mujeres remarcaron la imposibilidad de decidir sobre los cuidados y las prácticas realizadas durante el parto o la cesárea. En algunos casos, las mujeres indicaron que los profesionales de la salud no habían respetado sus decisiones, forzándolas a recibir prácticas que ellas habían rechazado expresamente: “Estresante, fue una cesárea y como me cubría una manta la mitad del cuerpo no sabía lo que sucedía. Lo más feo fue que al sacar al bebe uno no puede ver lo que le están haciendo los doctores antes de mostrarlo a la madre" (Caso 11), "fue una angustia saber que me iban a cesarear y ni me daban explicación del por qué" (Caso 12), “LO QUE HICIERON FUE COMUNICAR TODO A MI ESPOSO QUE ESTUVO PRESENTE, NO SENTI QUE ME PIDIERON CONSENTIMIENTO” (Caso 8), ${ }^{15}$ “Un poco traumática, porque tuve que pelear con la anestesia para no dormirme" (Caso 6). 


\section{Valoración global de la experiencia del parto}

El 29 por ciento de las participantes en este estudio valoraron su experiencia de parto o cesárea como buena o muy buena y el 33 por ciento la valoró como negativa o muy negativa. Una mujer mostró indicios de sufrir trastorno de estrés postraumático: "la experiencia que tuve con la cesárea no me gusto creo que fue más tenso, doloroso y traumático tanto que me da temor tener otro bebé" (Caso 13).

El 29 por ciento de las mujeres evitó ofrecer una valoración abiertamente positiva o negativa. La metodología utilizada en esta investigación no permite comprender por qué estas mujeres no pudieron realizar una valoración firme sobre su experiencia de parto o cesárea.

Figura 5

VALORACIÓN GLOBAL DE LA EXPERIENCIA DEL PARTO

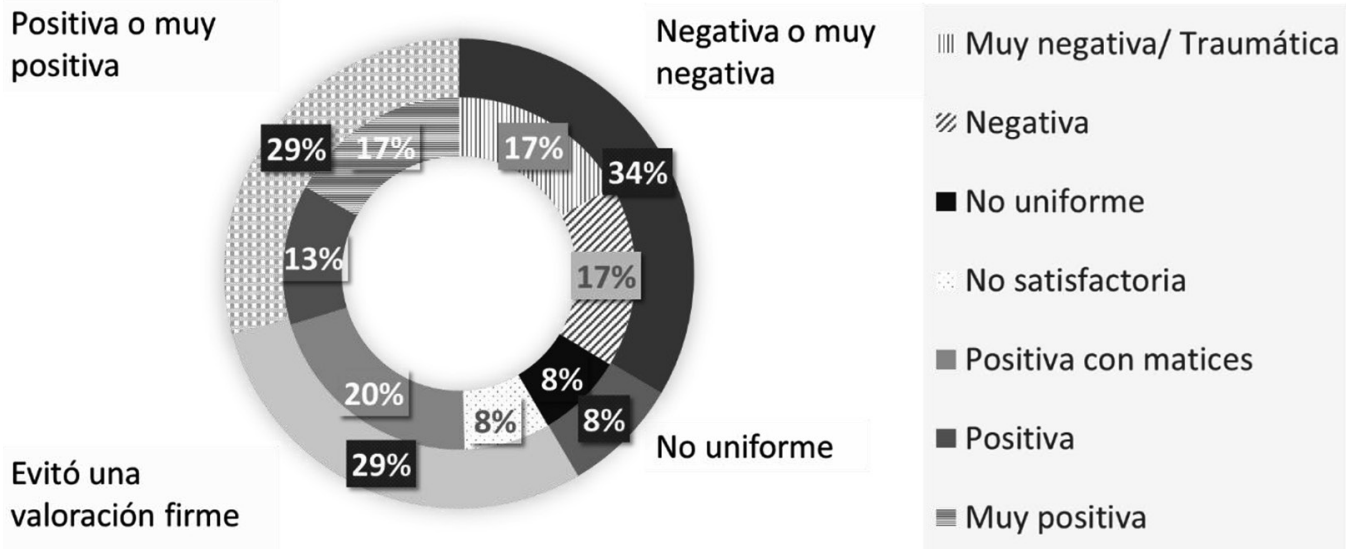

Fuente: Elaboración propia a partir de los datos del estudio.

Por último, el 8 por ciento de las mujeres (2 mujeres) declaró que la experiencia fue irregular a lo largo de las fases del parto, siendo en un caso positiva durante la fase de dilatación y negativa durante el expulsivo, y viceversa en el otro caso.

\section{Conclusiones}

La sobretasa de cesáreas es un problema público de naturaleza multicausal vinculado con la existencia de factores que afectan gravemente al ejercicio de DSR de las mujeres.

Los resultados de esta investigación exploratoria hablan de un cumplimiento claramente insatisfactorio de las recomendaciones para los cuidados al parto emitidas 
por la Declaración de Fortaleza en 1985 y por la OMS en 1996. De los 11 indicadores analizados, solo uno se cumplió satisfactoriamente y solo dos presentaron un desfase entre el estándar y el valor muestral menor al 25 por ciento.

Las mujeres que participaron en este estudio señalaron que el contacto piel con piel (entendido como la no separación de la criatura recién nacida), el apoyo afectivo y el respeto al consentimiento informado eran cuidados de gran relevancia para vivir una experiencia positiva durante el parto o la cesárea.

Los datos recogidos en relación a la falta de aplicación del consentimiento informado y a la realización de tactos vaginales traumáticos y de episiotomías rutinarias, nos indican que la violencia obstétrica fue habitual en la atención al parto de estas mujeres. Destaca asimismo la gran cantidad de mujeres que realizaron el expulsivo en posición de litotomía, que es en principio la posición menos propicia para parir. Ello hace sospechar sobre la existencia de restricciones importantes a la agencia de las mujeres para decidir en qué postura quieren parir, y sobre la extensión del control que el personal de salud realiza sobre el parto, a pesar de que ser este un proceso fisiológico propio de las mujeres.

Finalmente, menos de un tercio de las mujeres de la muestra calificaron su experiencia de parto como buena o muy buena.

Los resultados de este estudio indican que para entender los motivos que subyacen bajo la sobretasa de cesáreas en Nicaragua, es necesario estudiar los factores que inciden en la toma de decisiones sobre la vía del nacimiento, incluyendo aquellos vinculados con los aspectos sociales, culturales y políticos de la atención al parto, además de los aspectos económicos y técnicos. Para ello, considero necesario llevar a cabo una investigación con una metodología mixta que permita determinar en qué medida los cuidados al parto en Nicaragua se apegan a las recomendaciones orientadas por la OMS y qué factores están limitando la calidad de estos cuidados. Los cuidados al parto deben ser examinados desde una perspectiva cualitativa -no solo cuantitativa- analizando cómo las políticas públicas y los distintos actores presentes en el parto (mujeres con experiencias de parto o cesárea, sus familias, redes comunitarias, personal de salud, instituciones hospitalarias y medios de comunicación, entre otros) reconstruyen o limitan los preceptos culturales que afectan a la toma de decisiones sobre los cuidados al parto y la vía de nacimiento.

Este estudio debe llevarse a cabo desde la óptica de la teoría del biopoder, según la cual la relación entre pacientes y personal médico se inscribe dentro de un sistema social relacional de poder particular en el que, para el caso de la atención al parto, participa una variable fundamental en el análisis sociopolítico: el género.

En conjunto, dada la relevancia de este problema de salud pública -en especial para el bienestar de las mujeres y las criaturas recién nacidas en su sentido más amplio-, su vinculación con la mortalidad y morbilidad materna y neonatal prevenible, y su impacto social a múltiples niveles al mermar el empoderamiento de las mujeres 
gestantes, se apela a la comunidad investigadora a abordar las barreras que a lo interno de cada país están limitando el acceso de las mujeres gestantes a cuidados de calidad. Los avances logrados en otros países en materia de DSR vinculados al parto, señalan que para lograr cambios significativos en esta área, es preciso que las mujeres y las comunidades participen en el sistema de salud reclamando su derecho a recibir cuidados de calidad durante el parto. Para coadyuvar en este proceso, es necesario que la comunidad investigadora contribuya a la construcción de otros imaginarios del parto, desgranando sus significados, y facilitando el acceso de los actores sociales a este conocimiento. Esta labor, necesaria pero no suficiente para lograr el cambio social que se precisa para que las mujeres accedan a sus derechos también durante el parto, permitiría a la ciudadanía imaginar otra realidad posible, y luchar por ella.

\section{Notas}

1 Este estudio no hubiera sido posible sin la participación de las mujeres que participaron facilitando sus experiencias de parto y sin el apoyo de la Lic. Rebeca Benavente durante la fase de campo.

2 Un avance de este estudio fue presentado dentro del XV Congreso Centroamericano de Sociología, celebrado entre el 12 y el 14 de octubre del 2016 por la Asociación Centroamericana de Sociología (ACAS), en la Universidad Centroamericana, en Managua (Nicaragua).

A través del Mapa de padecimientos de salud de Nicaragua, publicado por el Ministerio de Salud de Nicaragua (http://mapasalud.minsa.gob.ni/mapa-de-padecimientos-de-salud-denicaragua), se pueden obtener cifras sobre las altas hospitalarias realizadas por "Parto por cesárea" y por "Parto" en el 2016 y 2017: para el año 2016 esta fuente indica que se dieron 74.892 altas hospitalarias por parto y 32.445 por parto por cesárea, y 96.935 altas hospitalarias por parto y 37.290 por parto por cesárea para el 2017. No he utilizado estos datos para calcular la tasa de cesáreas pues considero que la información que los acompaña es insuficiente para construir una metodología de cálculo rigurosa. Por ejemplo, no es posible identificar si las mujeres que fueron dadas de alta por "Parto prematuro, trabajo de parto prolongado y hemorragia postparto" (la tercera causa de hospitalización de las mujeres embarazadas) o "Embarazo múltiple, desproporción cefalopélvica y polihidramnios" (séptima causa) tuvieron un parto vaginal o un nacimiento por cesárea.

4 Las estadísticas de ENDESA 2011/2012 (elaborada por el Instituto Nacional de Información de Desarrollo (INIDE)), nos dicen que por tipo de proveedor, la tasa promedio más alta de cesáreas para el periodo 2006-2012 se produjo en los hospitales privados, donde se situó en el 78 por ciento de los partos totales.

En el apartado 34 del Consenso de Montevideo sobre Población y Desarrollo, los DSR se describen como aquellos que permiten vivir una sexualidad plena en condiciones seguras, incluyendo el derecho a tomar decisiones libres, informadas, voluntarias y responsables sobre la sexualidad (Comisión Económica para América Latina y el Caribe (CEPAL) y Organización de las Naciones Unidas (ONU)).

La maniobra de Kristeller consiste en "realizar presión sobre el fondo uterino durante el expulsivo, bien con una mano, dos o el antebrazo, con el fin de acortar la duración del mismo" 
expulsando el feto al exterior (Asociación el parto es nuestro 2012). Por su parte, la maniobra de Hamilton se realiza con el fin de madurar el cuello del útero para provocar el inicio del trabajo de parto y consiste en introducir un dedo dentro del orificio del cuello del útero, realizando un giro completo sobre la base del útero con el fin de despegar las membranas de la bolsa amniótica de la base del útero (González-Boubeta y Cid-González 27).

También conocida como postura ginecológica.

Hospital público con servicios de referencia nacional en salud materna. El Hospital Bertha Calderón forma parte de la red de establecimientos del MINSA y atiende a población del régimen no contributivo.

Estos organismos son: National Institute for Health and Care Excellence (NICE), Royal College of Obstetricians and Gynaecologists (RCOG), American College of Obstetricians and Gynecologists (ACOG) y el National Institutes of Health (NIH).

En la actualidad, el PVDC está contraindicado para las mujeres que han sufrido rotura uterina previa o con una cicatriz de cesárea clásica o vertical, y en cualquier caso para las mujeres con otras contraindicaciones absolutas para el parto, como la posición fetal transversa o la placenta previa total, las cuales se aplican independientemente de la presencia de una cicatriz uterina (RCOG 2015 6).

Ya en 1996 la OMS consideraba que la rotura rutinaria de membranas amnióticas era una práctica sobre la que no existía una evidencia clara para sostener su aplicación y que la estimulación con oxitocina sintética se utilizaba inadecuadamente $(2015$ 17, 20).

En la actualidad, la necesidad de realizar episiotomías, aún de forma selectiva, es muy controversial. Algunos estudios indican que esta debe realizarse bajo las siguientes indicaciones: parto complicado (parto de nalgas, sospecha de posible distocia de hombro), parto instrumental, cicatrices de mutilación de los genitales femeninos o de desgarros de tercer o cuarto grado mal cicatrizados y riesgo de pérdida del bienestar fetal (Molina-Reyes 103).

El artículo 9.1 de la CDN reconoce que “Los Estados Partes velarán por que el niño no sea separado de sus padres contra la voluntad de éstos, excepto cuando, a reserva de revisión judicial, las autoridades competentes determinen, de conformidad con la ley y los procedimientos aplicables, que tal separación es necesaria en el interés superior del niño" (Unicef Comité español).

En la muestra estudiada, la maniobra de Kristeller se le realizó al 33 por ciento de las mujeres con experiencia de parto y con cesárea urgente.

Se respeta el uso de las mayúsculas efectuado por la mujer que aportó este testimonio.

\section{Bibliografía}

Alba, Concepción et al. Maternidad y Salud. Ciencia, Conciencia y Experiencia. Madrid: Ministerio de Sanidad, Servicios Sociales e Igualdad, 2012.

INSS. Anuario Estadístico 2014. Managua: INSS, 2015. https://www.inss.gob.ni/images/anuario_estdistico_2014.pdf

INIDE. Anuario Estadístico 2015. Managua: INIDE, 2016. http://www.inide.gob.ni/Anuarios/ Anuario\%20Estadistico\%202015.pdf 
Arrieta, Alejandro y Andrés Oneto. “Quiénes ganan y quiénes pierden con los partos por cesáreas?" Proyecto Mediano. Auspiciado por El Programa Investigación ACDI-DRC, 2007.

Asociación el parto es nuestro. "Dossier STOP Kristeller: fundamentación jurídica”. El parto es nuestro. https://www.elpartoesnuestro.es/informacion/dossier-stop-kristeller-fundamentacion-juridica

Asociación el parto es nuestro. “Campaña de información y sensibilización sobre la maniobra de Kristeller". El parto es nuestro, diciembre del 2012. https://www.elpartoesnuestro. es/informacion/campana-de-informacion-y-sensibilizacion-sobre-la-maniobra-dekristeller

Cabral, José Augusto. “New tendencies in medicalization”. Ciência \& Saúde Coletiva, 13 (2008): 579-587.

Cabral, José Augusto. "Pensando o processo saúde doença: a que responde o modelo biomédico". Saúde e sociedade, 11.1 (2002): 67-84.

Campos, Paula. "Colegio De Matronas: "La violencia obstétrica existe, pero la ejerce el Estado'"'. Diario Uchile, 12 de octubre del 2014. http://radio.uchile.cl/2014/10/12/ colegio-de-matronas-la-violencia-obstetrica-existe-pero-la-ejerce-el-estado/

Caño, África et al. Papel de la matrona en la disminución de la tasa de cesáreas. Hospital Universitario Virgen de las Nieves. Granada, 2017. Recuperado de http://www.hvn. es/servicios_asistenciales/ginecologia_y_obstetricia/ficheros/actividad_docente_e_ investigadora/i_jornada_partos/8_papel_matrona_disminucion_cesareas.pdf

Carroli, Guillermo y Luciano Mignini. “Episiotomy for Vaginal Birth.” The Cochrane database of systematic reviews, 1 (2009): CD000081. PMC. 17 de julio del 2018. http://cochranelibrary-wiley.com/doi/10.1002/14651858.CD000081.pub2/full

CEPAL, NU. Consenso Montevideo sobre población y desarrollo. CELADE, septiembre del 2013. Conferencia Regional sobre Población y Desarrollo de América Latina y el Caribe. http://repositorio.cepal.org/bitstream/handle/11362/21835/4/S20131037_es.pdf

Coalition for Improving Maternity Services. The Risks of Cesarean Section: A Coalition For Improving Maternity Services Fact Sheet. Raleigh, 2010. http://www.motherfriendly.org/ resources/documents/therisksofcesareansectionfebruary2010.pdf

Colomar, Mercedes et al. "Mode Childbirth Low Risk Pregnancies Nicaraguan Physicians 'Viewpoints'”. Maternal child health journal, 18.10 (2014): 2382-2392.

Cunningham, F. Gary et al. Williams Obstetrics. Brasil: McGraw Hill, 2014.

Curran, Eileen A., et al. "Association between obstetric mode of delivery and autism spectrum disorder: a population-based sibling design study". JAMA psychiatry, 72.9 (2015): 935-942. https://archpsyc.jamanetwork.com/article.aspx?doi=10.1001/jamapsychiatry.2015.0846

Dekker, Rebecca. "Friedman's Curve Failure Progress: A Leading Cause Unplanned C-sections". Evidence Based Birth, 28 de agosto del 2013. https://evidencebasedbirth.com/friedmans-curve-and-failure-to-progress-a-leading-cause-of-unplanned-c-sections /

Delgado, Janet. “Vulnerabilidad y fragilidad. La importancia de los vínculos”. Bioética De La Maternidad. Humanización, Comunicación Y Entorno Sanitario (1. ${ }^{a}$ ed.). Margarita Boladeras Cucurella y Josefina Goberna Tricas (Coords.). Barcelona: Edicions de la Universitat de Barcelona, 2017. 91-100. www.publicacions.ub.edu/ejecuta_descarga.asp ?codart $=08628 \& c c=\& m p=43$ L41o31B75I \&formato $=$ PDF 
Delgado, Janet. "Autonomía relacional. Un nuevo enfoque para La bioética”. Tesis de maestría. Universidad de Educación a Distancia, 2012. e-spacio.uned.es/fez/eserv/bibliuned:m asterFilosofiaFilosofiaPractica-Jdelgado/Documento.pdf

INIDE. Encuesta Nicaragüense Demografía y Salud 2011/12. Managua: INIDE, 2013. www.inide. gob.ni/imagesnew/checkmark.gif

Federación de Asociaciones de Matronas de España. "La dilatación. Prácticas habituales". Iniciativa Parto Normal. Documento de consenso. Barcelona: Federación de Asociaciones de Matronas de España, 2007. 35-44. www.federacion-matronas.org/ipn/documentos/ iniciativa-parto-normal?currentitemid $=3788$

Feito, Lydia. "El concepto de la autonomía en la medicina occidental". Bioètica E debat: tribuna abierta Institut Borja de Bioètica, 17.62 (2011): 1-6.

Fernández, Francisca. “¿Qué es La violencia Obstétrica? Algunos aspectos sociales, éticos y jurídicos". Dilemata, 18 (2015): 113-128.

González-Boubeta, Roberto y Carla Cid-González. “Maduración cervical: aceleración de un proceso natural". Matronas Profesión, 8.1 (2007): 24-29.

Gibbons, Luz et al. "The global Numbers costs additionally needed unnecessary caesarean sections performed year overuse barrier universal coverage". World health Report, 30 (2010): 1-31.

Hartmann, Katherine et al. "Outcomes routine episiotomy. Systematic review". Jama, 293.17 (2005): 2141-2148.

Hodges, Susan. "Abuse hospital-based birth settings?" The Journal perinatal education, 18.4 (2009): 8.

"Pasos para ser IHAN- Hospitales. 10 PASOS EN HOSPITALES". iHan, www.ihan.es/quees-ihan/pasos/pasos-para-ser-ihan-hospitales/. Fecha de último acceso: 18 de julio del 2018

Landy, Helain J. et al. "Characteristics associated with severe perineal and cervical lacerations during vaginal delivery". Obstetrics and gynecology, 117.3 (2011): 627.

Nicaragua. Ley No.423, Ley General Salud. Managua: Gaceta Diario Oficial 91 (2002): 3473-3488. http://deviunn.asamblea.gob.ni/iunp/docspdf/gacetas/2002/5/g91.pdf

"Mapa de padecimientos de salud de Nicaragua". Mapasalud.minsa.gob.ni. http://mapasalud. minsa.gob.ni/mapa-de-padecimientos-de-salud-de-nicaragua. Fecha de último acceso: 24 de febrero del 2018.

Massó, Ester. "DESEO LACTANTE: Sexualidad y política en el lactivismo contemporáneo". Antropología Experimental, 13 (2013): 515-529.

Ministerio de Salud de Nicaragua. Normativa 109, Protocolo para La Atención las Complicaciones Obstétricas. Managua: MINSA, 2013.

Ministerio de Salud de Nicaragua. Normativa 108, Guía Clínica Para La Atención Del Neonato. Managua: MINSA, 2013.

Ministerio de Salud de Nicaragua. Normativa 077, Protocolo para El abordaje las patologías más frecuentes del Alto Riesgo Obstétrico. Managua: Gobierno de Reconciliación y Unidad Nacional, 2011.

Ministerio de Salud de Nicaragua. Normativa 042, Norma Humanización del Parto Institucional. Managua: MINSA, 2010.

Ministerio de Salud de Nicaragua. Normativa 030, Guías Cuidado Enfermería. Embarazo, Parto, Puerperio y Recién Nacido. Managua: GRUN, 2010. 
Molina-Reyes, Cristina, et al. "Implantación de una política de episiotomía selectiva en el Hospital de Baza. Resultados materno-fetales". Progresos de Obstetricia y Ginecología, 54.3 (2011): 101-108.

Narváez, Alexis Rafael. “Indicaciones cesárea en el Hospital Bertha Calderón Roque, Managua año 2014". 2015.

Odent, Michel. "El nacimiento los mamíferos humanos". Obstare El mundo la maternidad, 15 (2004): 23-29.

Odent, Michel. "La visión de la investigación en la salud primal". I Congreso Internacional de Parto y Nacimiento en Casa. Jerez de la Frontera: Asociación Nacer en Casa, 2010: 56-58. http://nacerencasa.org/i-congreso-internacional-de-parto-y-nacimiento-en-casa-jerez-de-la-frontera-2000

Olza, Ibone. "PTSD obstetric violence." Midwifery today international midwife, 105 (2013): 48-9.

OMS. Cuidados en el parto normal. Una guía práctica. Ginebra: OMS, 1996.

OMS. "Recomendaciones la OMS sobre el nacimiento: Declaración Fortaleza. Tecnología Apropiada para El parto". Lancet, 2 (1985).

OMS. Prevención y erradicación la falta respeto y el maltrato durante la atención del parto en centros de salud. Ginebra: OMS, 2014.

OMS. Recomendaciones de la OMS para la conducción del trabajo de parto. Ginebra: OMS, 2015.

OMS. Declaración de la OMS sobre tasas de cesárea. Ginebra: Organización Mundial de la Salud, 2015. http://apps.who.int/iris/bitstream/10665/161444/1/WHO_RHR_15.02_spa.pdf

Palomo, María Teresa. "El 'care', un debate abierto: de las políticas de tiempos al 'social care'". Cuestiones de género: de la igualdad y la diferencia, 4 (2009): 325-355.

Poblete, Margarita y Sandra Valenzuela. “Cuidado humanizado: un desafío para las enfermeras en los servicios hospitalarios". Acta Paul Enferm, 20.4 (2007): 499-503.

Polidano, Cain, Anna Zhu y Joel C. Bornstein. "The relation between cesarean birth and child cognitive development". Scientific reports, 7.1 (2017): 11483. https://www.nature.com/ articles/s41598-017-10831-y

Gupta, JK, GCS Smith y RR Chodankar. "Birth after previous caesarean birth” Greentop guideline, 45. Londres: Royal College of Obstetricians and Gynaecologists RCOG, 2015. www.rcog.org.uk/womens-health/clinical-guidance/birth-afterprevious-caesareanbirth-green-top-45

Sadler, Michelle, María Elena Acuña y Alexandra Obach. Nacer, Educar, Sanar; Miradas desde la Antropología del Género. Catalonia, 2004.

Sáez, Cristina. “Congreso en Barcelona. Los niños nacidos por cesárea tienen más riesgo de alergias". La Vanguardia. 15 de marzo de 2015. www.lavanguardia.com/ciencia/20150315/54428157306/cesarea-alergias-microbiota-asma-lactancia-materna.html

Federación Internacional de Planificación de la Familia. “Derechos Sexuales. Una declaración IPPF." Londres: Federación Internacional de Planificación de la Familia, octubre del 2008.

UNICEF Comité Español. Convención sobre los Derechos del Niño. Madrid: UNICEF Comité Español, junio del 2006.

Lumbiganon, Pisake et al. "Method delivery Pregnancy outcomes Asia WHO global Survey maternalPerinatalhealth2007-08." Lancet, 375.9713 (2010): 490-499. www.thelancet. com/journals/lancet/article/PIIS0140-6736(09)61870-5/fulltext 
Villaverde, María Victoria. “Variaciones en la utilización de la cesárea en los hospitales públicos del Sistema Nacional de Salud". Tesis doctoral. Universidad de Zaragoza, 2009. zaguan.unizar.es/record/5233/files/TESIS-2010-051.pdf

Wagner, Marsden. "La visión de la investigación en la salud primal". I Congreso Internacional de Parto y Nacimiento en Casa. Jerez de la Frontera: Asociación Nacer en Casa, 2010: 6-10. http://nacerencasa.org/i-congreso-internacional-de-parto-y-nacimiento-en-casajerez-de-la-frontera-2000

María Augusta Rodrigues Ribeiro. Investigadora social de origen hispanoportugués, formada como economista especializada en género, desarrollo humano y desarrollo socioeconómico. Actualmente, realiza un doctorado en el Programa de Estudios Interdisciplinares de Género de la Universidad Autónoma de Madrid (España). Ha colaborado con la Universidad Centroamericana en el campus de Managua (Nicaragua) en la docencia de análisis y evaluación de políticas públicas y de género y equidad social. Como consultora independiente, ha prestado servicios especializados en el ciclo de gestión de proyectos y programas de intervención social a instituciones del gobierno de Nicaragua, organismos de cooperación gubernamental, organizaciones del tercer sector y Organizaciones de la Sociedad Civil (OSC).

Contacto: mar.rodrigues01@predoc.uam.es

ORCID: 0000-0002-5753-4631 
